\title{
Separating damage from environmental effects affecting civil structures for near real-time damage detection
}

\author{
William Soo Lon Wah, Yung-Tsang Chen, Gethin Wyn Roberts and \\ Ahmed Elamin
}

\begin{abstract}
Analyzing changes in vibration properties (e.g. natural frequencies) of structures as a result of damage has been heavily used by researchers for damage detection of civil structures. These changes, however, are not only caused by damage of the structural components, but they are also affected by the varying environmental conditions the structures are faced with, such as the temperature change, which limits the use of most damage detection methods presented in the literature that did not account for these effects. In this article, a damage detection method capable of distinguishing between the effects of damage and of the changing environmental conditions affecting damage sensitivity features is proposed. This method eliminates the need to form the baseline of the undamaged structure using damage sensitivity features obtained from a wide range of environmental conditions, as conventionally has been done, and utilizes features from two extreme and opposite environmental conditions as baselines. Toallow near real-time monitoring, subsequent measurements are added one at a time to the baseline to create new data sets. Principal component analysis is then introduced for processing each data set so that patterns can be extracted and damage can be distinguished from environmental effects. The proposed method is tested using a two-dimensional truss structure and validated using measurements from the Z24 Bridge which was monitored for nearly a year, with damage scenarios applied to it near the end of the monitoring period. The results demonstrate the robustness of the proposed method for damage detection under changing environmental conditions. The method also works despite the nonlinear effects produced by environmental conditions on damage sensitivity features. Moreover, since each measurement is allowed to be analyzed one at a time, near real-time monitoring is possible. Damage progression can also be given from the method which makes it advantageous for damage evolution monitoring.
\end{abstract}

Keywords

Damage detection, environmental conditions, temperature variation, principal component analysis, structural health monitoring, data normalization

\section{Introduction}

Civil structures, such as bridges and buildings, are in constant degradation due to the severe environmental and operational conditions they are faced with. To provide a reliable and safe society, a robust structural health monitoring (SHM) system is required to monitor the health conditions of the structures. It could allow the authorities to take appropriate measures to repair or replace defect structural components before catastrophic failures occur. As a result, the safety and lifetime of the structures could be increased.
During the past decades, researchers have been active in developing methods for damage detection of civil structures, and these methods are mainly based on

Department of Civil Engineering, The University of Nottingham Ningbo China, Ningbo, P.R. China

Corresponding author:

Yung-Tsang Chen, Department of Civil Engineering, The University of Nottingham Ningbo China, 199 Taikang East Road, Ningbo 315100, P.R. China.

Email: Yung-Tsang.Chen@nottingham.edu.cn 
analyzing the vibration properties (e.g. natural frequencies) of the structures which change when damages occur. However, even though these techniques have been tested and validated using numerical and experimental model structures, difficulties have arisen in implementing them for real-time monitoring of inservice structures. The primary reason behind this is that the vibration properties used as damage sensitivity features in the developed damage detection methods are also affected by the changes occurring in the environmental conditions (e.g. ambient temperature) the structures are faced with, which were not considered in most of the developed methods. ${ }^{1}$

In the literature, it was found that, for bridge structures, temperature plays a major role in the variability occurring in the vibration properties of the bridges..$^{2-7}$ It was reported that temperature may cause up to 5\%$10 \%$ variation in the natural frequencies of highway bridges, which unfortunately can mask the changes produced by structural damage. ${ }^{8}$ For example, for the Alamosa Canyon Bridge, Farrar et al. ${ }^{2}$ found out that the first natural frequency fluctuated by about $5 \%$ over a monitoring period of $24 \mathrm{~h}$. Similar variation in natural frequency was also observed in other modes of vibration of the bridge. These variations in natural frequencies were found to have a clear correlation with the temperature differentials across the bridge's deck. Similarly, Alampalli ${ }^{3}$ reported that the changes in natural frequencies of an abandoned bridge caused by freezing support were greater than those due to the effects of damage. Moreover, Peeters and De Roeck ${ }^{4}$ found out that temperature had a major influence on the natural frequencies of the Z24 Bridge in Switzerland. When the bridge was in its undamaged condition, a bilinear relationship was found for most combinations of natural frequency versus temperature (temperature of deck soffit, temperature of wearing surface, etc.). The authors concluded that the nonlinear relationship was attributed to the asphalt layer which, at temperature below $0^{8} \mathrm{C}$, contributed to the increase in stiffness of the structure, while at warmer temperature, it did not have an influence.

The variability occurring in the vibration properties of bridge structures due to temperature may be attributed to several factors. These factors have been discussed by Zhou and $\mathrm{Yi}^{9}$ and are summarized below. For example, the shear modulus and Young's modulus of the bridges' materials, which mainly determine the vibration properties of the structures, vary with the fluctuation of temperature. The stiffness of the supports and expansion joints of the bridges may also change due to the daily and seasonal temperature variations, which as a result, weaken or strengthen their constraints. These modifications may then change the vibration properties of the structures. Also, the sizes of the bridges' elements change because of thermal contraction and expansion due to temperature variations. This, therefore, changes the physical parameters of bridges and hence the vibration properties. Moreover, the temperature across the whole structure is usually non-uniform and changes with time, which, as a result, produces an asynchronous change in the physical parameters of the structure, thus contributing to the changes in vibration properties. Finally, thermal stresses induced by temperature and the stress redistribution in the structures also affect the vibration properties of structures.

Due to the contributing factors mentioned above that affect the vibration properties of structures, the use of these properties as damage sensitivity features for damage detection is restrained because false damage alerts may occur. Hence, to provide a robust and reliable damage detection method which does not give false damage alerts due to the effects of the changing environmental conditions, these effects should be taken into account while deriving the method. In the literature, dealing with these effects for damage detection is often referred to as data normalization problem. ${ }^{1}$ One approach used extensively in data normalization is to perform regression analysis between the damage sensitivity features (e.g. natural frequencies) and their corresponding environmental parameters (e.g. temperature) in which they were obtained. ${ }^{4,10-14}$ This approach creates a model that can predict the values of damage sensitivity features given the conditions the structures are faced with. Any large error can then be attributed to damage. To use this approach, however, the baseline of the undamaged structure should be composed of damage sensitivity features obtained from a wide range of environmental conditions, so that it covers all possible scenarios the structure may encounter. The damage sensitivity features and their corresponding environmental conditions should also be measured for each observation which, therefore, introduces some practical difficulties. For example, although it is easy to measure the damage sensitivity features and their corresponding environmental parameters, the optimal locations to place the sensors can be difficult to determine and to emplace. ${ }^{15}$ Also, after the baseline has been created, the sensors must remain at the same locations on the structure. ${ }^{16}$ Any failure occurring in any of the sensors may affect the performance of the SHM system. Moreover, since each structure is different, difficulties will arise in choosing which environmental parameters most affect the damage sensitivity features to measure, since past experience of other structures cannot be reliably used. Due to these aforementioned constraints, the use of this approach for data normalization is therefore limited.

Another common approach that has been proposed in the literature is to extract features that are sensitive 
to damage but less sensitive to the effects of the changing environmental conditions. ${ }^{15,17-19}$ It has the advantage over the previous approach in that measurements of the environmental parameters are not required; only the damage sensitivity features are needed. Among the numerous techniques adopted in this approach, principal component analysis (PCA) has been widely used. For example, Manson ${ }^{20}$ proposed to perform PCA on the damaged sensitivity features data set and to perform an outlier analysis on the last principal components to indicate the presence of damage. The principle behind the method is that the first principal components, which contain most of the variances in the data set, will account for the effects of the changing environmental conditions affecting the data set, while the last principal components will account for other effects of the likes of damage. Therefore, by analyzing only the last principal components, the effects of environmental conditions affecting the data set can be eliminated while the effects of damage are retained. Similar to Manson, ${ }^{20}$ Yan et al. ${ }^{16}$ also used the concept that the effects of environmental conditions and the effects of structural damage will be accounted for by different principal components. They proposed to retain and use the higher variance principal components as a model to reconstruct the original data set so that the minor factors of the likes of damage affecting the data set can be eliminated. Then, by subtracting the newly formed data set from the original data set, a residual error can be obtained and be used to indicate damage. Any large residual error can then be attributed to the presence of damage.

Since PCA is a linear analysis tool and the effects of environmental conditions on the damage sensitivity features are usually nonlinear, Yan et al. ${ }^{21}$ further extended the previous method to take into account the nonlinear effects. They proposed to first cluster the data set into several linear data sets, followed by analyzing each linear data set similar to the linear case. As a result, the nonlinear system is linearized. Sohn et al. ${ }^{22}$ proposed the use of auto-associative neural network to perform nonlinear principal component analysis (NLPCA). The NLPCA is trained using the autoassociative neural network to extract the dependency of the damage sensitivity features on the environmental conditions. The auto-associative neural network used in the NLPCA consists of three hidden layers with the first being the mapping layer, the second being the bottleneck layer, and the third being the de-mapping layer. The unmeasured environmental conditions are represented in the bottleneck layer. The target output is equal to the input which is the damage sensitivity features. Damage is indicated when the prediction error increases. They proposed to obtain the damage sensitivity features through the use of auto-regressive and auto-regressive with exogenous inputs (AR-ARX) model. Reynders et al. ${ }^{23}$ proposed to use kernel PCA to create a nonlinear output-only model of the undamaged structure to be used as baseline. New measurements can be compared to the model, and any growth in the prediction error can then be attributed to damage.

For all the methods mentioned above, however, as for the regression analysis approach, it is important to capture the damage sensitivity features from a wide range of environmental conditions so that the baseline can cover all possible scenarios the structure may encounter. Therefore, developing a method that does not require the damage sensitivity features to be obtained from a wide range of environmental conditions will make the SHM system easier to construct. Also, using only damage sensitivity features and removing the needs to measure the environmental parameters the structures are faced with after the baseline has been formulated, will make the damage detection method less prone to failure. Furthermore, developing a method that can analyze one time measurement at a time without requiring a group of measurements for analysis is a way forward for real-time monitoring of structures.

In this article, a damage detection method for data normalization that does not require the baseline to consist of damage sensitivity features obtained from a wide range of environmental conditions is proposed. Since usually some environmental parameters are measured, to make full use of them, some of the parameters are used to help in the creation of the baseline. In the proposed method, the baseline consists of damage sensitivity features obtained at two extreme and opposite environmental conditions. Subsequent measurements can then be added to the baseline one at a time to form new data sets to be analyzed separately for damage detection. PCA is proposed to be used to process each new data set to distinguish between damage effects and environmental effects affecting the damage sensitivity features. Analyzing one data set at a time allows the proposed method to perform near real-time monitoring of structures. It is worth noting that after the baseline has been created, the environmental measurements are not required anymore and only the damage sensitivity features are needed for future analysis. The proposed method uses a two-dimensional (2D) truss structure model subjected to a varying temperature to simulate a varying environmental condition and the Z24 Bridge in Switzerland to test and validate its robustness. The results demonstrate the effectiveness of the proposed method in distinguishing between the effects of damage and the effects of the changing environmental conditions affecting damage sensitivity features through the use of principal components obtained by applying 
PCA. The method is also proved to be suitable for

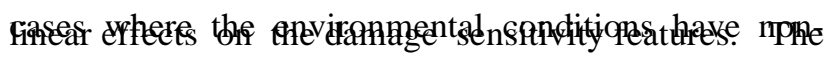

robustness of using PCA in the proposed method to analyze real-life structures is shown with the high successful rates obtained in classifying the undamaged and damaged cases of the Z24 Bridge.

The remainder of the article first starts with the presentation of the methodology used, with an in-depth description of the proposed method, given. Then, in section "Case studies," the two case studies are described with the results and analysis given altogether. Finally, section "Conclusion" concludes the article.

\section{Methodology}

This section introduces the proposed damage detection method to distinguish damage effects from environmental effects affecting damage sensitivity features of structures. First, a brief introduction on PCA, which is used for data processing, is given along with a visualization interpretation on how PCA can be used in the context of this study. Then, the damage detection method is described in detail, followed by a summary of the procedures in order to implement the proposed method given to conclude this section.

\section{PCA}

PCA is a multivariate statistical technique used to highlight the similarities and differences in a data set by finding patterns in the set. It is mainly used to reduce the dimensions of the original data set without losing much of the information. ${ }^{24}$ It forms new variables representing the factors which most characterize the variances in the original data set using linear combination of each of the original variables. ${ }^{25}$

Damage sensitivity features collected from civil structures subjected to varying environmental conditions can be processed using PCA to extract the main factors driving the variances in the data set. These factors may be due to the varying environmental conditions of the likes of ambient temperature as well as to damage of structural components. A brief description on the principle behind PCA is given below.

Let $\mathrm{Z}$ denote a $\mathrm{n} 3 \mathrm{p}$ data set of damage sensitivity features collected from $\mathrm{p}$ observations with $\mathrm{n} \backslash \mathrm{p}$. For each observation, $n$ numbers of damage sensitivity features are collected. If, for example, natural frequencies of the structure are chosen as the features, then $n$ represents the number of frequencies selected and $p$ represents the amount of time the natural frequencies are collected

$$
\begin{array}{rlr}
{ }_{\mathrm{z}_{1,1}} & \cdots & \mathrm{z}_{1, \mathrm{p}} \\
\mathrm{Z}=6_{\mathrm{z}_{\mathrm{n}, 1}} & \cdots & \mathrm{z}_{\mathrm{n}, \mathrm{p}}
\end{array}
$$

To perform PCA on the damage sensitivity features data set, mean centering of the data set is first required. This is achieved by subtracting the mean of each row of the data set to each measurement in that row. The resulting matrix $X$ after mean centering will have the same dimensions (n 3 p) as the original data set $\mathrm{Z}$. Note that performing data centering does not change the relative location of each point to each other; it only changes the mean to zero and the centroid location to zero coordinates.

PCA transforms the data set $X$ into a new $\mathrm{m} 3 \mathrm{p}$ data set $Y$ with smaller dimensions which characterizes most of the variances in the original data set. The relationship between $\mathrm{Y}$ and $\mathrm{X}$ can be expressed using a transformation matrix $T$ which has dimensions $\mathrm{m} 3 \mathrm{n}$ as follows

$$
\mathrm{Y}=\mathrm{TX}
$$

$\mathrm{Y}$, the newly formed data set, is called the score matrix. It is a new set of data which combines the scores of each observation obtained for different factors affecting the original data set into a matrix. The factors here are called principal components and they may represent the environmental effects as well as damage of structural components affecting the damage sensitivity features. The principal components are formed in such a way that the first principal component accounts for most of the variances in the original data set and the second component accounts for the second most variances, and so on. The principal components in the matrix $\mathrm{Y}$ are arranged in descending order, with the first principal component representing the factor(s) producing the greatest variances in the original data set, while the last principal component representing the factor(s) producing the least variances. The score of each observation for each principal component can be thought of as a coordinate along the principal component axis representing the location of each observation along each axis. The scores for the first principal component will have the greatest span over the axis, whereas the last principal component will have the smallest span. PCA makes the first principal component to have the greatest span by rotating the cloud of data in such a way to minimize the distance of each point in the cloud to the first principal component axis while assuring that the axis goes through the zero centroid. It is for this reason that PCA requires mean centering of the original data set prior to application. 
In mathematical term, the transformation matrix $\mathrm{T}$ is called the loading matrix, and it contains coefficients which are used to compute the score matrix through linear combinations of the variables in the data set X. The rows of the loading matrix $\mathrm{T}$ correspond to the eigenvectors of the covariance matrix of $X$, and they can be obtained by decomposing the matrix $\mathrm{X}$ using singular value decomposition and use that decomposition to construct the covariance matrix of $\mathrm{X}$ as follows

$$
\frac{1}{p-1} \mathrm{XX}^{\mathrm{T}}=\mathrm{U} \frac{\mathrm{S}^{2}}{\mathrm{p}-1} \mathrm{U}^{\mathrm{T}}
$$

where $\mathrm{U}$ is an orthonormal matrix $\left(\mathrm{UU}^{\mathrm{T}}=\mathrm{I}\right)$ whose columns represent the eigenvectors of the covariance matrix of $\mathrm{X}$ (hence $\mathrm{T}=\mathrm{U}^{\mathrm{T}}$ ), and $\mathrm{S}$ is given as

$$
\mathrm{S}=\begin{array}{cc}
\mathrm{S}_{1} & 0^{1} \\
0 & \mathrm{~S}_{2}
\end{array}
$$

with the diagonal terms being represented by the singular values $S_{1}=\operatorname{diag}\left(S_{1}, S_{2}, \ldots, S_{m}\right)$ and $S_{2}=\operatorname{diag}\left(S_{m}+1\right.$,

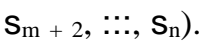

The singular values $S_{1}$ and $S_{2}$ are arranged in descending order $\left(\begin{array}{llllll}\mathrm{S}_{1} & \mathrm{~S}_{2} & \ldots & \mathrm{S}_{\mathrm{m}} & \mathrm{S}_{\mathrm{m}+1} & \ldots \ldots \ldots \ldots . . .\end{array}\right.$ $S_{n}$. Each singular value indicates how much variance its corresponding eigenvector explains in the original data set. Therefore, $S_{1}$ represents factors that have the largest influence on the original data set (e.g. the effects of the changing environmental conditions and the effects of damage), while $S_{2}$ represents the factors with the least effects on the original data set (e.g. noise). Since $S_{2}$ can be very small and close to zero, it is usually discarded to reduce the dimensions of the original data set. If the contribution of all the eigenvectors are added together, they will explain all the variances in the original data set (i.e. $100 \%$ in terms of percentage information of the original data set). Since each principal component has a corresponding eigenvector from which they are calculated, to know the percentage of variance (information) each principal component describes, the eigenvalue of that principal component can be divided by the summation of all the eigenvalues which represent the total information of the original data set.

By applying equation (2), the score matrix $\mathrm{Y}$ can be generated. Usually, to reduce the dimensions of the original data set, only the first $m$ rows (eigenvectors) of the loading matrix $\mathrm{T}$ are used to construct the score matrix. However, in this study, all the rows are kept to construct the score matrix, since in this study, PCA is used to extract the similarities and differences in the original data set rather than reducing the dimensions of the original data set. Analyzing only the first few rows of the score matrix (first few principal components), damage detection can be performed. It should be noted that the number of principal components to be analyzed should be chosen carefully so that false alarms do not occur. An approach to choose the number of principal components to be analyzed will be given later in this section, with an in-depth explanation behind the concept given in section "Case studies,", so that illustrations and examples can be used for better understanding.

\section{Visual interpretation}

To illustrate how PCA can be used to distinguish between the effects of damage and the effects of changing environmental conditions affecting damage sensitivity features of structures, a visual interpretation is given here. Consider the data set given in Table 1, which represents the performance of four students in eleven tests. The rows represent the marks (maximum $100 \%$ ) obtained by each student in a particular test, while the columns represent the marks obtained by the individual student in each test. For this case, the students represent the different observations (i.e. $p=4$ ), while the tests represent the different variables (i.e. $\mathrm{n}=$ 11) affecting the data set (i.e. data set $Z$ ). For this illustrative example, the number of variables is more than the number of observations ( $n$ - p). Therefore, there will only be $\mathrm{p}-1$ number of eigenvectors, hence, only $\mathrm{p}-1$ number of principal components. Having only $\mathrm{p}+$ principal components instead of $\mathrm{n}$ components is not of great importance because when applying PCA with the proposed damage detection method, only the first few principal components which have a major contribution on the data being analyzed will be examined. Thus, even though this illustration is not of the same dimensions as the data set of damage sensitivity features (i.e. damage sensitivity features data set with dimensions $\mathrm{n} \backslash \mathrm{p}$ having $\mathrm{n}$ principal components) that will be analyzed, it will not affect the principle behind the approach adopted due to the fact that only the first

Table 1. Test results for the four students.

\begin{tabular}{lllll}
\hline Test no. & Student A & Student B & Student C & Student D \\
\hline 1 & 70 & 71 & 71 & 73 \\
2 & 65 & 63 & 64 & 65 \\
3 & 75 & 77 & 75 & 76 \\
4 & 80 & 81 & 79 & 82 \\
5 & 79 & 82 & 80 & 80 \\
6 & 70 & 70 & 65 & 72 \\
7 & 71 & 70 & 64 & 71 \\
8 & 80 & 82 & 75 & 80 \\
9 & 60 & 61 & 60 & 60 \\
10 & 65 & 67 & 70 & 65 \\
11 & 85 & 87 & 85 & 86 \\
\hline
\end{tabular}




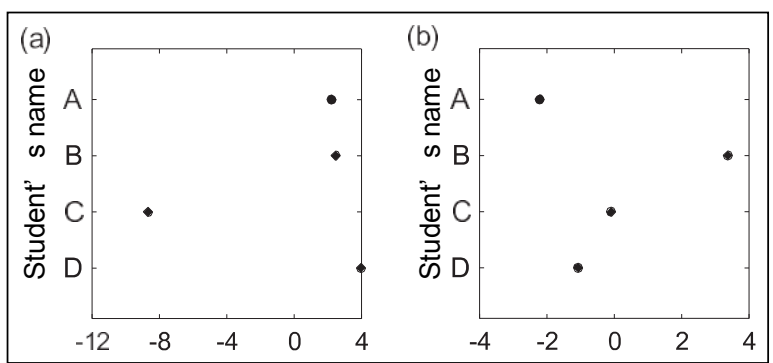

Figure 1. Graph of (a) first principal component and (b) second principal component.

few principal components will be examined and the last few principal components which have almost no influence on the data set will not be investigated.

Looking at the data alone, it is difficult to extract useful information on how each student compares with each other; therefore, by applying PCA on the data set, common patterns can be extracted to show the students with similar performances and to highlight those who performed differently. Figure 1 gives the plots of the first and second principal components for all four students. It can be seen from plot in Figure 1(b) that no useful information on which student is outlier from the rest can be extracted since the scores of the students are well distributed throughout that component. However, from plot Figure 1(a), a clear distinction can be seen between student $\mathrm{C}$ and the remaining students. Student $\mathrm{C}$ seems to perform differently from the rest of the students. Looking back at the data set given in Table 1 , it can be seen that student $\mathrm{C}$ performs better in test 10 than in tests 6 and 7 while students A, B, and D perform better in tests 6 and 7 than in test 10 . From Figure 1, it is evident that by applying PCA, the patterns in the data set can be extracted and important information on how the students compare can be obtained. Thus, similar to this example, it is expected that damage effects can be distinguished from environmental effects affecting damage sensitivity features of structures for damage detection.

\section{Damage detection method}

As shown previously, PCA can be used to extract patterns in a data set. When PCA is applied, the first principal component will represent the factor(s) that creates most of the variances in the original data set, the second principal component for the factor(s) that creates the second most variances, and so on. Therefore, to enable the first principal component to represent the effects of the changing environmental conditions affecting the damage sensitivity features, data from two extreme and opposite environmental conditions (e.g. temperature of
$-30^{8} \mathrm{C}$ and $70^{8} \mathrm{C}$ ) can be used to enhance the variability in the data set caused by the environmental effects. As a result, the plot representing the environmental factor will have the greatest span and hence largest variance. Each extreme case will be at the opposite side of each other on the first principal component plot. Generally, only one or two environmental conditions will have a large effect on the damage sensitivity features of structures; therefore, the first principal component alone is generally enough to represent most of the variances in the data set. The other components will represent other minor factors of the likes of noise affecting the damage sensitivity features of the structure.

If data from an observation gathered at a normal environmental condition (e.g. temperature at $20^{8} \mathrm{C}$ ) from the undamaged structure is added to the two extreme and opposite cases data set and PCA is performed, the score of this observation will lie in between the two extreme cases in the first principal component plot. For this undamaged structure, the first principal component alone will account for most of the information of the data set since only the environmental effects that have a consequent influence on the data set. Now, if data gathered from a damage state structure at a normal environmental condition is added to the two extreme cases and PCA is applied, the score for that damage state will also lie in between the two extreme cases in the first principal component plot since that component represents the environmental effects. However, unlike the undamaged state, for this damage state, the second principal component will also represent a significant amount of the information of the data set. This second principal component will represent the undamaged and damaged effects affecting the structure. In that second component, the two extreme cases will cluster together with a separation between them observed and will be at the opposite end to the damage state observation score on the plot since the undamaged structure and the damaged structure have completely different behavior from each other. This approach can thus be used to distinguish between damage and environmental effects affecting damage sensitivity features of structures. The only requirement is that a baseline consisting of damage sensitivity features obtained at two extreme and opposite environmental conditions needs to be created. Subsequent measurements can then be added to the baseline one at a time to create different data sets, and PCA be performed on each set for data processing. Analyzing the plots of the first few principal components, damage effects can then be differentiated from environmental effects. The undamaged structure will lie in between the two extreme cases, while the damaged structure will lie outside the baseline. Thus, the indicator of the presence of damage for this proposed damage detection method is 


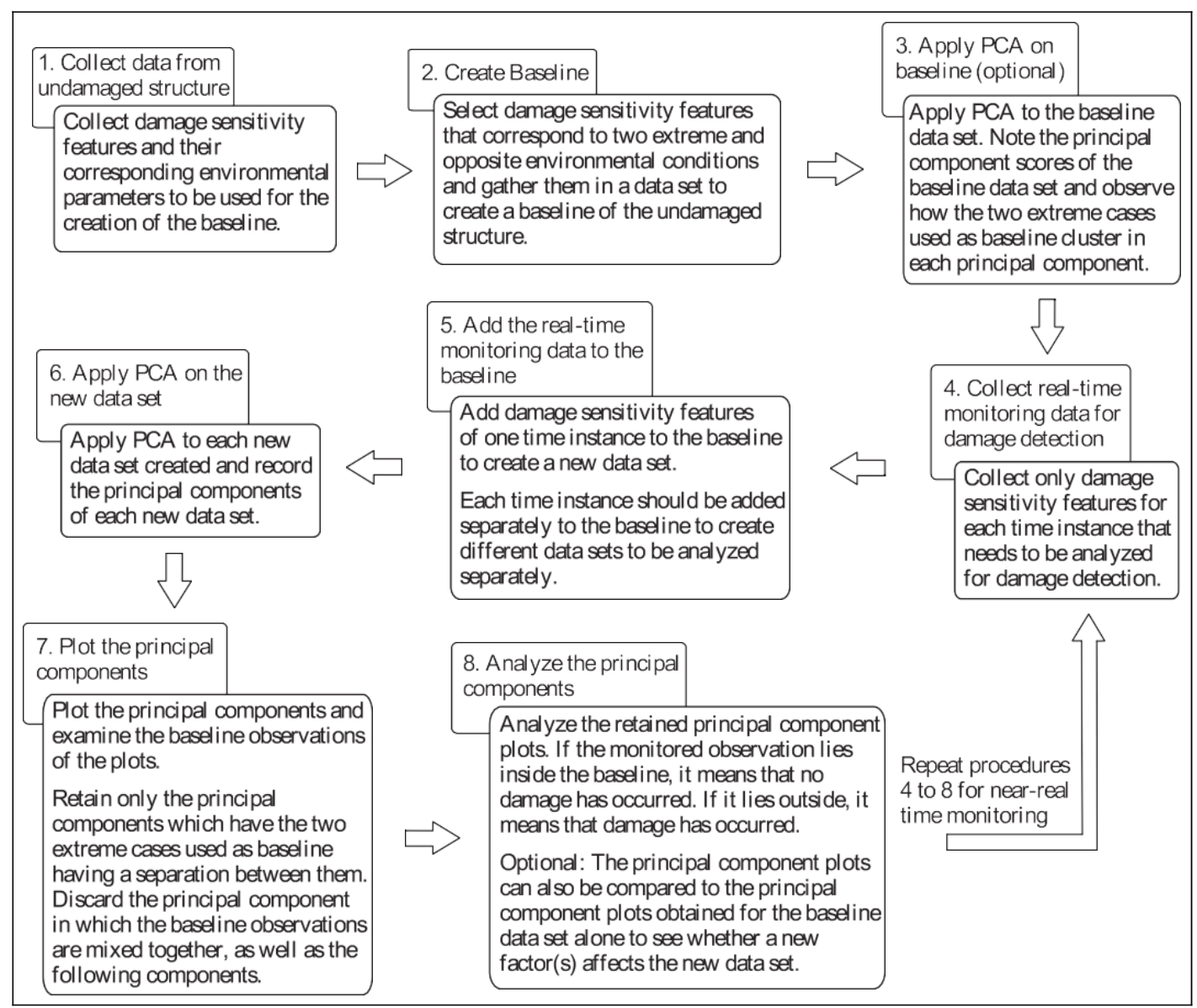

Figure 2. Procedures to follow for damage detection.

when the monitored observation lies outside the two extreme and opposite conditions used as baseline for the undamaged structure.

As mentioned previously, the number of principal components to analyze needs to be chosen carefully to avoid false alerts. As a general rule, the principal components that have the baseline scores obtained from the two extreme cases to have a separation between them should be analyzed for damage detection. The principal component in which the two baseline cases are mixed together should be discarded as well as the following components. The reason behind this is that in the creation of the baseline, two extreme and opposite cases have been selected on purpose so that they have a separation between them to indicate two different behaviors obtained by the effects of the same factor(s). More information on that will be given in the next section so that illustrative examples can be used for better understanding.

\section{Damage detection procedure}

A description of the procedures to follow to implement the proposed damage detection method is given in
Figure 2. Procedures $4-8$ should be repeated to analyze new measurements for near real-time monitoring. Procedure 3 and the last part of Procedure 8 are optional since they are not compulsory for the implementation of the proposed method. They are here to help distinguish whether a new factor that did not affect the baseline data set affects the monitored cases.

\section{Case studies}

To illustrate and test the proposed damage detection method, two cases are considered in this section. A numerical truss structure model, which is subjected to a varying temperature to simulate the effects of a varying environmental condition, is first analyzed. Then, the method is applied to the Z24 Bridge, which was monitored for nearly one year with realistic damage scenarios introduced to the bridge near the end of the monitoring period.

\section{Simulated truss structure model}

The finite element model of the 2D truss structure shown in Figure 3 consists of 30 elements. The 


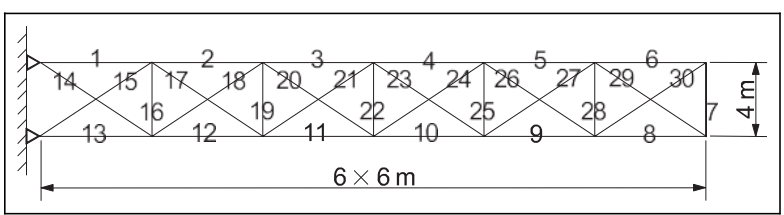

Figure 3. Two-dimensional truss structure model.

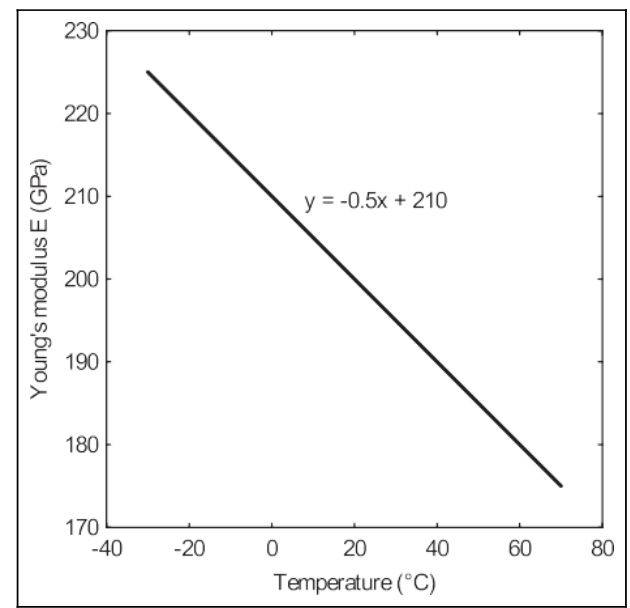

Figure 4. Graph of Young's modulus versus temperature.

structure is pin-connected to a wall and all elements are pin-connected to each other. All the members are assumed to be made with the same steel material with Young's modulus of $200 \mathrm{GPa}$, density of $7850 \mathrm{~kg} / \mathrm{m}^{3}$, and cross-sectional area of $0.001 \mathrm{~m}^{2}$. Only one environmental condition is assumed to be applied to the structure, which is a varying temperature condition. To simulate the varying temperature condition, Young's modulus is assumed to be temperature dependent (Figure 4) with reference temperature taken at 208C. The range of temperature considered in this study is

$-108 \mathrm{C}$ to $408 \mathrm{C}$. The temperature across the whole structure is assumed to be the same. It is assumed that the first four natural frequencies of the structure are readily available to be used as damage sensitivity features. Damage in the structure is assumed to be represented by reductions in axial stiffness of the truss members.

A plot of the variations of natural frequencies with temperature is given in Figure 5. It can be seen that the natural frequencies decrease with an increase in temperature. Hence, the use of natural frequencies as damage sensitivity features can give rise to false damage alerts if the effects of the temperature change are not considered. Thus, the proposed method is applied to distinguish between damage effects and temperature effects affecting the natural frequencies of the truss structure.

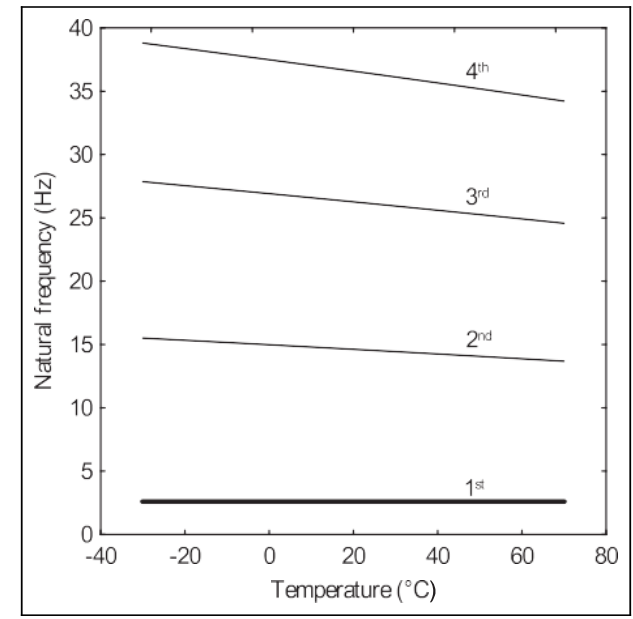

Figure 5. Variations of natural frequencies with temperature.

Table 2. Description of undamaged and damaged cases.

\begin{tabular}{llll}
\hline Case & Temperature (8C) & Element & Extent (\%) \\
\hline Undamaged 1 & -10 & - & - \\
Undamaged 2 & 10 & - & - \\
Undamaged 3 & 20 & - & - \\
Undamaged 4 & 40 & - & - \\
Damaged 1 & 0 & 8 & 10 \\
Damaged 2 & 40 & 8 & 10 \\
Damaged 3 & 0 & 8 & 5 \\
Damaged 4 & -10 & 14 and 30 & 5 and 5 \\
\hline
\end{tabular}

Four undamaged and four damaged cases subjected to varying temperature are used to test the robustness of the proposed method. The descriptions of the cases are given in Table 2. To further illustrate, for example, for the first undamaged case, it is assumed that the temperature of the structure is at $408 \mathrm{C}$, while for the first damaged case, the temperature is assumed to be at $08 \mathrm{C}$ with element 8 having a damage extent of $10 \%$ (reduction in stiffness).

To distinguish damage effects from temperature effects affecting the damage sensitivity features of the truss structure, the baseline of the undamaged structure should consist of natural frequencies of the structure obtained at two extreme and opposite temperature con-

ditions. For a better performance of the proposed method, the baseline is made to consist of 10 extreme cases with five cases taken at temperatures of $-268 \mathrm{C}$ to $-308 \mathrm{C}$ and five cases at temperatures of $668 \mathrm{C}$ to $708 \mathrm{C}$ with $18 \mathrm{C}$ interval. Five cases at low temperature and five cases at high temperature are chosen so that the relationship between the natural frequencies and the low and high temperatures can be extracted.

For the method to perform near real-time monitoring, each new observation to be monitored should be 
added to the baseline one at a time to create new data sets and PCA be applied on each new data set for processing. Each new data set $\mathrm{Z}$ will be a matrix of dimensions 4311 with the 4 rows corresponding to the first four natural frequencies of the structure, while the 11 columns to the 10 baseline observations plus the new observation which needs to be analyzed

$$
\begin{aligned}
& \begin{array}{lllll}
2 & \mathrm{f}_{1,1} & \cdots & \mathrm{f}_{1,10} & \mathrm{f}_{1, \mathrm{ob}}
\end{array} \\
& \mathrm{Z}=\begin{array}{llll}
\mathrm{f}_{2,1} & \cdots & \mathrm{f}_{2,10} & \mathrm{f}_{2, \text { ob }} \\
4 \mathrm{f}_{3,1} & \cdots & \mathrm{f}_{3,10} & \mathrm{f}_{3, \mathrm{o}}
\end{array} \\
& \begin{array}{llll}
\hline \mathrm{f}_{4,1} & \cdots & \mathrm{f}_{4,10} & \mathrm{f}_{4, \mathrm{ob}}
\end{array}
\end{aligned}
$$

where $f_{i j}$ means the ith frequency from the jth observation; ob represents the new observation that needs to be monitored.

Since temperature is the only environmental parameter considered here and two extreme and opposite cases have been considered, as a result, the temperature effects will be represented by the first principal component. This is because the first principal component which accounts for most of the variances in the original data set has been forced to account for the effects of the varying temperature by selecting two extreme and opposite temperature conditions which will have completely opposite behavior to each other. The following principal components will account for the other factors of the likes of damage affecting the data set. The results of the four undamaged cases are first presented in Figure 6. Note that only the plots of the first two principal components are given in Figure 6. For all the cases, only the first principal component is retained for analysis for damage detection since only in that component the low and high temperatures baseline observations have a separation between them. The first 10 observations (dots) on the plots represent the two extreme cases used as baseline, while the eleventh observation (cross) is the monitored case that needs to be analyzed.

From the first principal component plots in Figure 6, it can be seen that for all the undamaged cases under the varying temperature condition, the proposed method does not raise the damage alert. All the monitored cases are between the two extreme and opposite cases, which indicates that the only consequent factor(s) affecting the natural frequencies of the structure is the temperature variation. It can therefore be concluded that the proposed method performs well for the undamaged cases subjected to varying temperatures. In addition to assessing the structural conditions of the structure, this method can also give a rough indication of the temperature condition the structure is faced with without directly recording the temperature measurement. From the first principal component plots in Figure 5, the evolution of temperature from undamaged case 1 -undamaged case 4 can be seen clearly. It
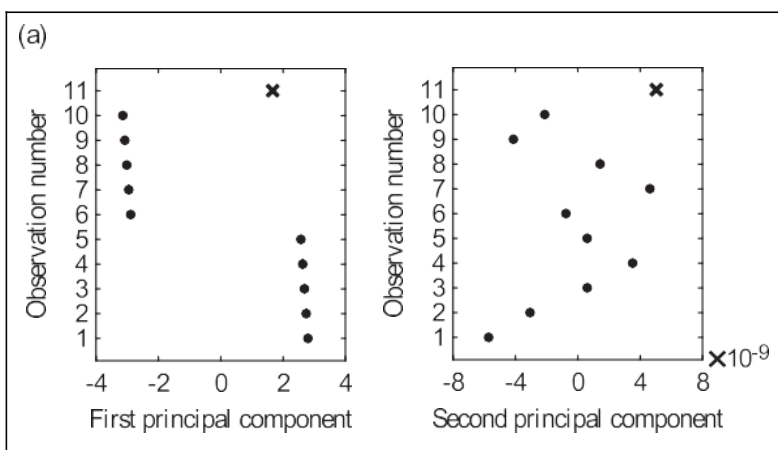

(b)
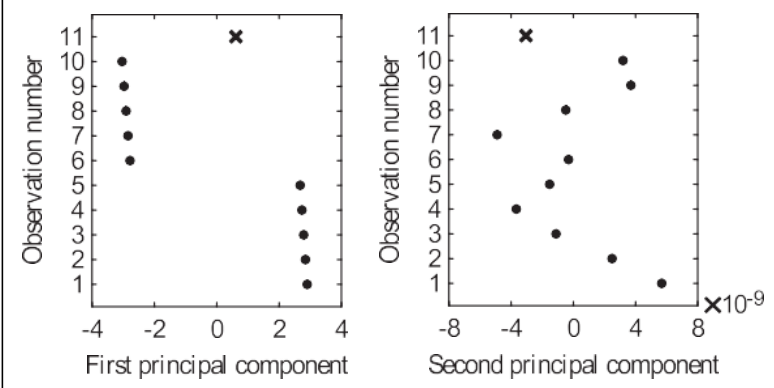

(c)
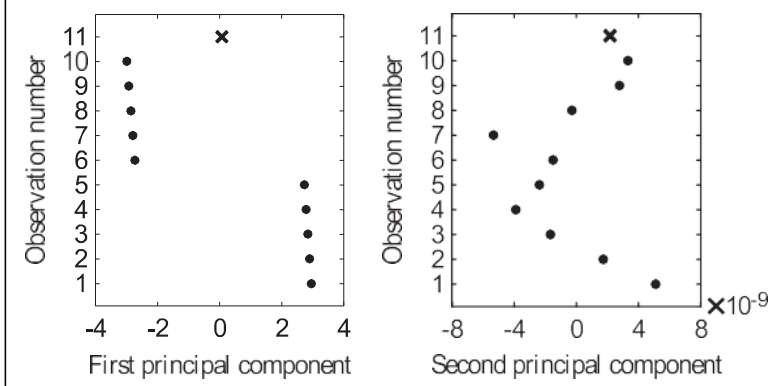

(d)
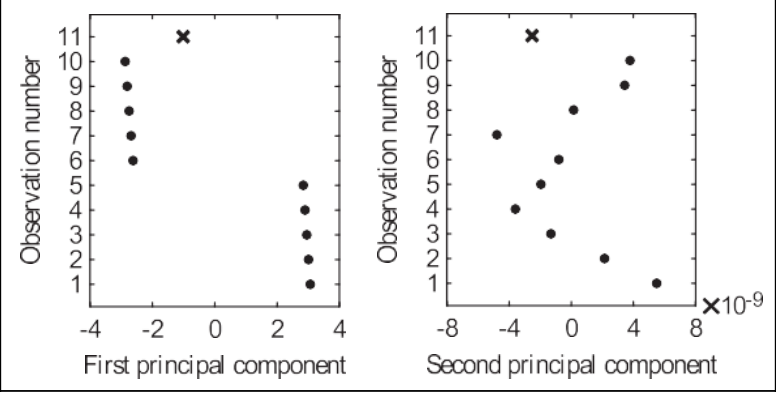

Figure 6. Graph of first and second principal components for undamaged (a) case 1, (b) case 2, (c) case 3 , and (d) case 4.

can be seen that the cross representing the monitored cases moves from one extreme temperature case (-308C) to the other extreme temperature case (70 8C) with increasing temperature.

It is interesting to note that the first principal component of the first undamaged case represents almost $100 \%$ of the information of the original data set, and the second principal component represents 1.49543 $10^{-16}(\%)$, while the rest of the components represent 
even less.For the other undamaged cases, the first principal component also represents almost $100 \%$ of the information of the original data set. Since the principal components are formed using the loading matrix, which is directly related to the eigenvectors and singular values of the covariance matrix of $\mathrm{X}$, those percentages are reflected on the magnitudes of the principal component scores. It can also be seen from Figure 6 that the magnitude of the observations in the first principal component plot is much larger than that in the second principal component plot for all four undamaged cases since the first principal component represents nearly $100 \%$ of the information of the original data sets, while the second component merely represents any information.

The principal components plots for the damaged cases in Table 2 are given in Figure 7. Since only the first two principal components have low- and hightemperature baseline observations not being mixed with each other, plots of only the first two principal components are given for each damaged case. The plots of the first principal component for all damaged cases show the monitored cases between the two extreme cases. Similar to the undamaged cases, this indicates that the temperature condition the structure is faced with is between the two extreme cases. The evolution of the temperature condition can also be seen from the first principal component plots. For example, it can be seen that the first principal component plots for damaged cases 1 and 3 are quite similar since both cases have the same temperature of $08 \mathrm{C}$. However, in damaged case 2, the monitored observation moves toward the hightemperature baseline since the temperature for that case is above $08 \mathrm{C}$; while in damaged case 4 , it moves toward the low temperature baseline since the temperature for that case is below $08 \mathrm{C}$.

From the second principal component plots in Figure 7, it can be seen that damage alert is raised for all the cases since the monitored cases are separated from the two extreme cases used as baseline. The two extreme cases cluster together on one side of the plot with a separation between them being observed, while the monitored cases are at the other end on the plot. This suggests that a factor other than the temperature variation has a consequent influence on the data set, and this is attributed to damage. Damage evolution from case to case can also be seen clearly from the plots. For example, for cases 1 and 3, which have the same temperature and same damage element but with different damage extent, the second principal component differs. The damaged case 3 has a smaller deviation from the baseline than damaged case 1 in the second principal component plot. This is because damaged case 3 has a smaller damage extent $(5 \%)$ than damaged case $1(10 \%)$, thus producing a smaller
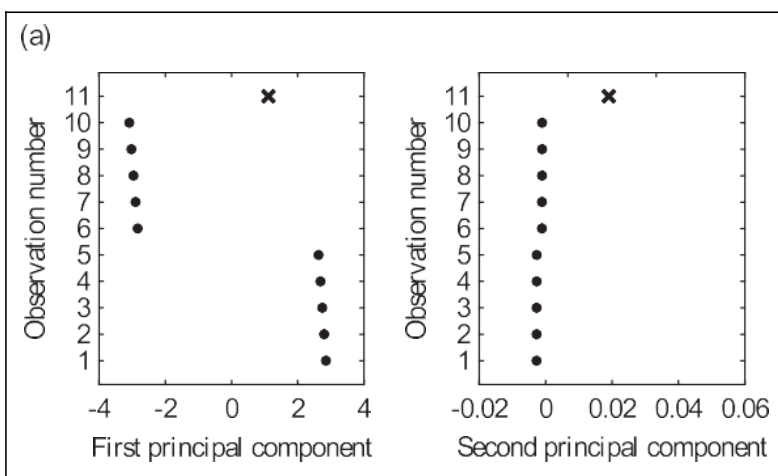

(b)
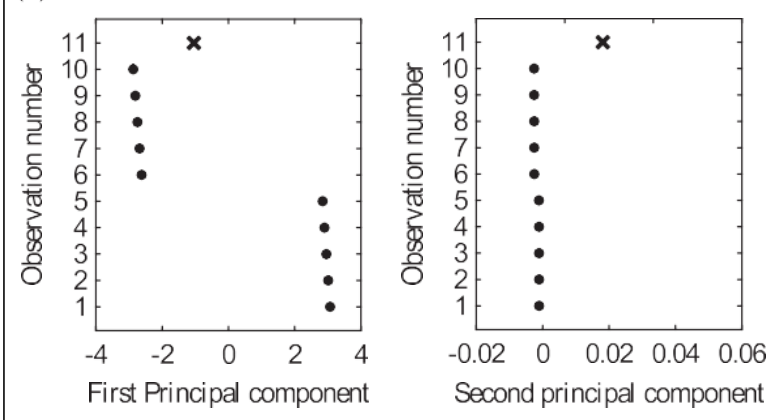

(c)
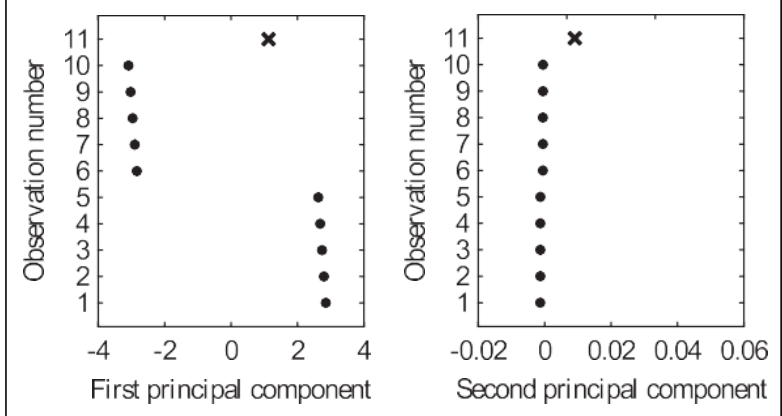

(d)
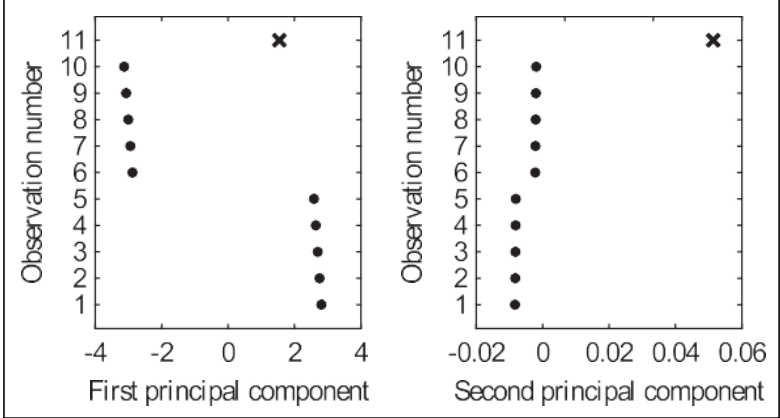

Figure 7. Graph of first and second principal components for damaged (a) case 1, (b) case 2, (c) case 3, and (d) case 4.

variance in the data set when compared to damaged case 1 . Therefore, from the results obtained, it can be concluded that the proposed method performs well in distinguishing damage from temperature effects affecting the natural frequencies of the structure. Small damages as well as multiple damages in the structure are 


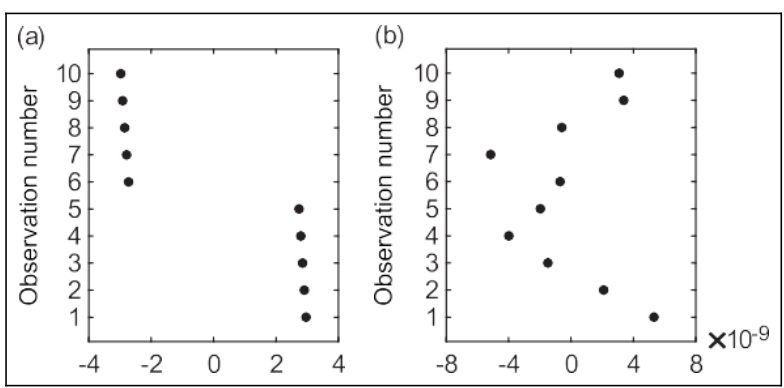

Figure 8. Graph of (a) first principal component and (b) second principal component for data set D1.

also well detected, which show the robustness of the proposed method.

For the damaged scenarios, it is worth noting that the first and second principal components, when combined together, contribute to almost 100\% (99.9995\% and $0.0005 \%$, respectively) of the information of the original data set. Also, the observations in the first principal component for the damaged cases have relatively the same magnitude as that of the undamaged cases. However, in the second principal component, the observations of the damaged cases have much higher magnitude than those of the undamaged cases, which therefore may indicate that another factor affecting the data set was not present in the undamaged cases.

\section{Anexplanation on the numberofprincipal components to analyze}

To select the number of principal components to analyze for damage detection, it may be tempting to choose those principal components that when summed up together describe almost $100 \%$ of information of the original data set. However, using this approach is subjective and can lead to false alerts. Generally, the environmental effects as well as damage effects will account for most of the information in the data set. However, other factors of the likes of noise and some nonlinear effects might also affect the data set greatly and these factors might account for some percentages of the information. Thus, analyzing these principal components which represent the noise effects and other nonlinear effects might lead to false alerts since these factors might affect the two extreme cases and the monitored case in a similar way, thus mixing and clustering them together in the principal component plots. As a result, false damage alerts may be raised in cases where the undamaged monitored cases lie outside the mixed baseline observations. Hence, a more practical approach to choose the number of principal components to analyze is also proposed in this article.
In the proposed approach, as mentioned previously, only the principal components that have the two extreme cases baseline observations to be separated from each other that should be analyzed for damage detection. The principal component that has baseline observations to be mixed together should be discarded as well as the following components. The reason behind this is that the baseline is made to consist of damage sensitivity features obtained at two extreme and opposite conditions so that for the same factor(s) (e.g. temperature and damage of structural components) affecting the original data set greatly, the two extreme cases will behave differently from each other, thus creating a distinction between them in the principal component plots. An illustrative example is given below using the data obtained from the truss structure case study for better understanding.

First, consider a data set D1 composed of the first four natural frequencies of the undamaged truss structure (Figure 3) used as baseline in the truss structure case study above (matrix $\mathrm{Z}$ with dimensions 4310 ). This data set D1 is also used as a baseline here for the proposed method. PCA is applied to the data set and the first and second principal component plots are given in Figure 8. In the first principal component plot (Figure 8(a)), the high-temperature measurements cluster together toward the left-hand side while the lowtemperature measurements cluster together on the righthand side of the plot as was meant to by choosing these two extreme and opposite temperature condi- tions. This separation between them indicates that the hightemperature measurements behave differently from the low-temperature measurements for that par- ticular principal component, and since it is known that two extreme and opposite temperature conditions have been chosen, it is concluded that the first principal component represents the temperature factor affecting the data set. In the second principal component plot (Figure 8(b)), the measurements from both temperature groups are mixed together and no clear distinction between them exists. This may be attributed to some factors that have the same influence on the high- and low-temperature measurements. It can also be seen that the magnitude of the observations in that component is relatively small when compared to the first principal component, which means that this factor does not influence the original data set greatly when compared to the temperature effects. Thus, it is proposed to analyze the first principal component only, since only in that component that a clear distinction between the two temperature groups can be seen, which was purposely designed for, through the two extreme cases adopted.

Now, consider another data set D2 with dimensions 4311 , composed of the natural frequencies of D1 used 


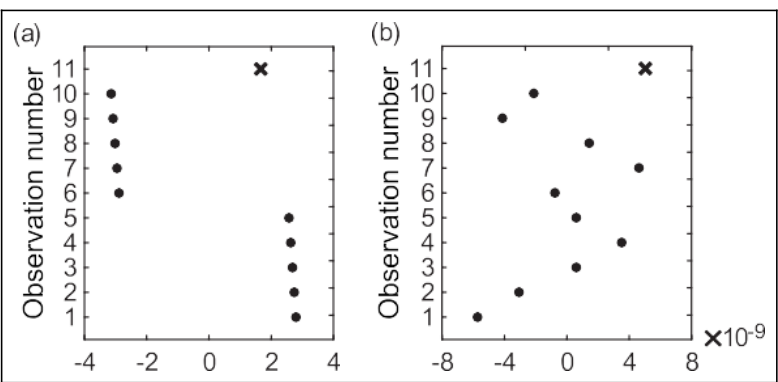

Figure 9. Graph of (a) first principal component and (b) second principal componentfor data set D2.

as baseline for damage detection plus another set of frequencies obtained from the undamaged case 1 in Table 2 used as the monitoring case. The first and second principal component plots for this data set are given in Figure 6(a). For convenience, the plots are given again here in Figure 9. From the first principal component plot, it can be seen that the low- and hightemperature baseline observations are well separated as was the case for data set D1. This, therefore, implies that this component represents the same factor as the first principal component of D1, which is the temperature factor. Moreover, in this plot, the observation that needs to be monitored lies between the baselines hence indicating that the temperature of this observation lies somewhere between the two extreme cases. In the second principal component plot, the baseline observations are mixed together and no separation can be seen between the high- and low-temperature measurements as was the case for data set D1. Furthermore, the magnitude of the observations for that component is relatively small, which indicates that this factor does not have a consequent influence on the data set. Hence, it can be concluded that this principal component does not need to be analyzed since the low- and hightemperature baselines are not separated from each other which was initially designed to be by using the two extreme cases. Thus, for data set D2, only the first principal component needs to be analyzed, and from the plot, it is concluded that the structure is undamaged.

It should be noted that if the second principal component plot of data set D2 is analyzed, damage alarm needs to be raised since the monitored case lies outside the baseline. This may be due to the fact that this second principal component affects the baseline and the monitored case in a similar way and hence there might be some cases where the monitored case will lie outside the baseline.

Figure 10 presents the first and second principal component plots of another data set D3, composed of

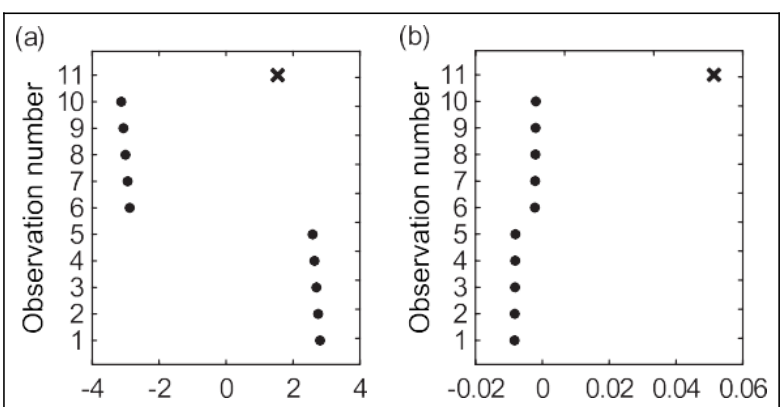

Figure 10. Graph of (a) first principal component and (b) second principal component for data set D3.

natural frequencies of D1 used as baseline for damage detection and damaged case 4 of Table 2 used for monitoring. These two plots are the same as those given in Figure $7(d)$. They are given again here for convenience since this damaged case is being used as an example to demonstrate how the principal components will behave under the presence of damage. The first principal component plot has the low- and high-temperature baselines being separated from each other similar to cases D1 and D2. This, therefore, indicates that this component represents the temperature effects affecting the data set. In the second principal component plot, a separation between the low- and high-temperature baselines can also be seen. In this plot, the two baseline cases cluster together on the right-hand side of the plot with a separation between them can be observed, while the monitored case is at the extreme other end of the plot on the left-hand side. When compared to the second principal component plots of data set D1 and D2, a distinct difference can be seen in the way the observations cluster as well as the magnitude of the observations in that principal component. Thus, this indicates that another factor(s) affects the data set D3 which did not affect the data sets D1 and D2. Since the baseline and the monitored case are at two ends and since the baseline cases are well separated, thus, it can be concluded that this component represents the undamaged and damaged effects affecting the structure. Hence, for this case, the first and second principal components should be analyzed for damage detection. From the second principal component plot, damage can be concluded to have occurred since the monitored case lies outside the baseline.

Figure 11 gives the third and fourth principal component plots for data set D3. They are given here to determine whether these components should be retained for analysis or they can be discarded. From the third principal component plot, it can be seen that all the baseline observations are mixed together. Thus, this 

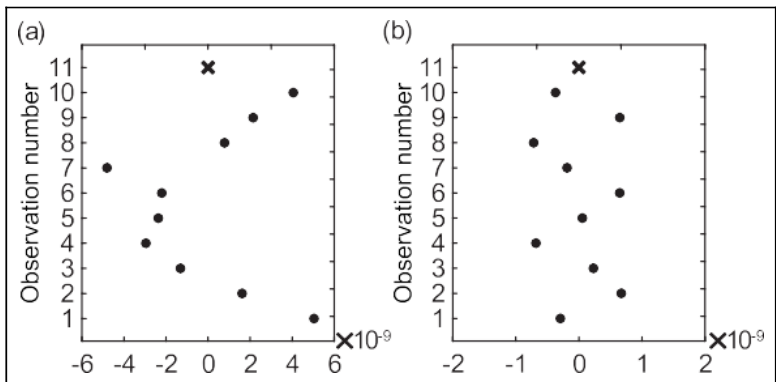

Figure 11. Graph of (a) third principal component and (b) fourth principal component for data set D3.

component as well as the following components should not be analyzed for damage detection.

To determine the number of principal components to analyze for damage detection, it is proposed to analyze only the plots where the two extreme and opposite cases used baseline have a separation between them. This can be achieved by plotting the first few principal components and discarding the principal component in which the two extreme cases used as baseline are mixed together as well as the following components. To get a better understanding of the factors that affect the data set as well as to make sure that the selected extreme cases baseline will be separated from each other after the application of PCA, it is suggested to apply PCA on the baseline data set alone first to get an overview of which principal components have the baseline being separated. Then, when applying PCA for monitoring, the results can be compared to the principal components obtained from the baseline data set alone to see whether they are similar, or whether a new factor(s) is affecting the data set. Usually, the first principal component will represent the effects of the selected environmental condition used to create the baseline since two extreme and opposite cases have been adopted, and the second principal component will represent the effects of damage since this is the second most consequent effect affecting the data set. However, there will be cases where the first principal component will represent the undamaged and damaged effects. This will be the case when damage in the structure is big enough to create a bigger variance in the data set than the two extreme and opposite environmental conditions used as baseline.

\section{Z24 Bridge}

The Z24 Bridge (Figure 12), a post-tensioned concrete box girder bridge, was located in Switzerland connecting Koppigen and Utzenstorf and overpassing the A1 highway between Bern and Zurich. It was a three-span

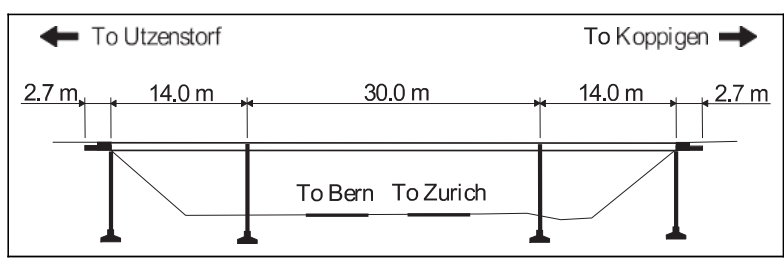

Figure 12. Z24 Bridge (Adapted from Peeters and De Roeck. ${ }^{4}$ )

Table 3. Description of the progressive damage cases applied to the Z24 Bridge.

\begin{tabular}{ll}
\hline Test no. & Case description \\
\hline 1 & Reference state \\
2 & System installation for piersettlement \\
3 & $20-\mathrm{mm}$ settlement of pier \\
4 & $40-\mathrm{mm}$ settlement of pier \\
5 & $80-\mathrm{mm}$ settlement of pier \\
6 & $95-\mathrm{mm}$ settlement of pier \\
7 & Foundation tilt \\
8 & New reference state \\
9 & $12 \mathrm{~m}^{2}$-chipping of concrete \\
10 & $24 \mathrm{~m}^{2}$-chipping of concrete \\
11 & Landslide \\
12 & Concrete hinges failure \\
13 & 2 anchorheads failure \\
14 & 4anchorheads failure \\
15 & Rupture of 2 tendons out of 16 \\
16 & Rupture of 4 tendons out of 16 \\
17 & Rupture of 6 tendons out of 16 \\
\hline
\end{tabular}

bridge with a main span of $30 \mathrm{~m}$ and two side spans of $14 \mathrm{~m}$ each. It was monitored for almost a year to collect different environmental parameters as well as acceleration measurements. The acceleration measurements were recorded for almost every hour and an automatic system identification system was in place to derive the modal parameters of the bridge. The Z24 Bridge was gradually damaged near the end of the monitoring period so that researchers could use the bridge as a benchmark structure to detect damages in the presence of the varying environmental conditions it was faced with.

The damaged cases that the bridge was subjected with are presented in Table 3. The damaged cases were selected so that they represented the possible damage scenarios that could arise to bridges during their lifetime. Therefore, this gives a good benchmark to test the proposed method to detect real damaged scenarios. Krämer et al. ${ }^{26}$ gave a detailed review of the damage cases introduced to the bridge.

The first four natural frequencies of the bridge along with several environmental parameters were made available to researchers. Therefore, the first four natural frequencies of the bridge are used as damage 


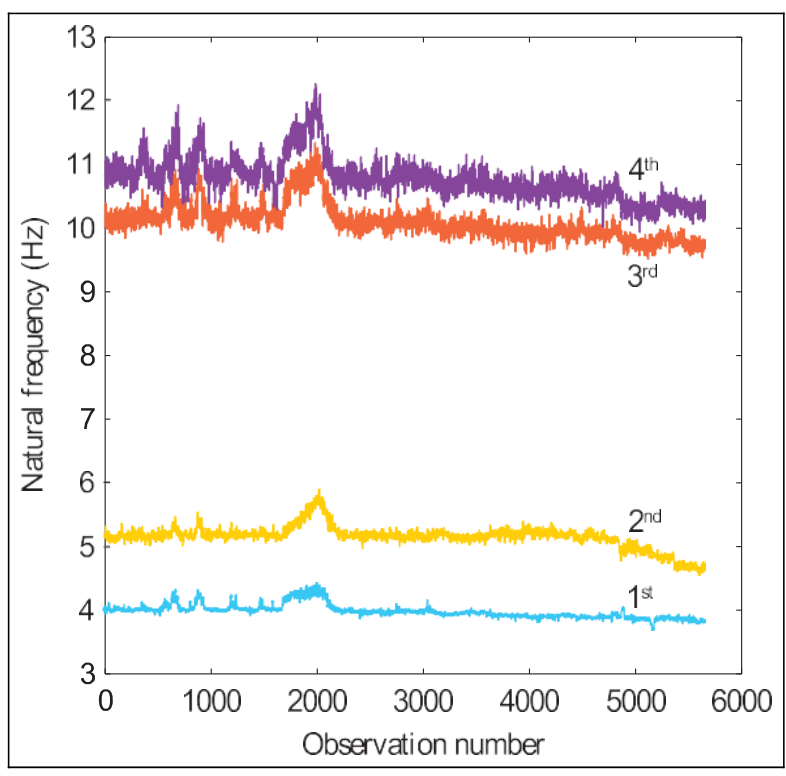

Figure 13. The variations of the first four natural frequencies over the whole monitoring period.

sensitivity features in this study. Figure 13 gives the plot of the variations of the first four natural frequencies of the bridge with time over the whole monitoring period. It can be seen that the natural frequencies fluctuate over time and analyzing the changes in natural frequencies without considering the environmental effects, at different time instance, cannot be used for damage detection. This would lead to false damage alerts. Using the proposed method presented in this article, damage detection under the varying environmental conditions can therefore be realized.

To perform the damage detection method proposed in this article, a baseline must first be established using natural frequencies obtained at two extreme and opposite environmental conditions. Since temperature had a major influence on the natural frequencies of the bridge, as was reported by Peeters and De Roeck, ${ }^{4}$ and since the temperature of the air is the environmental parameter that affects the rest of the temperature of the structure, it is chosen as the key parameter to help in the creation of the baseline. A bilinear relationship can be found between the temperature of the air and the first four natural frequencies of the bridge as shown in Figure 14 (graph only for monitored cases before damage was introduced). Peeters and De Roeck ${ }^{4}$ suggested that the bilinear relationship is attributed to the asphalt layer which, at temperature below $08 \mathrm{C}$, contributed to the increase in stiffness of the structure while at warmer temperature, it did not have an influence. These, therefore, affected the vibration properties differently. Consequently, the baseline should consist of natural frequencies obtained at temperatures below $08 \mathrm{C}$ and at
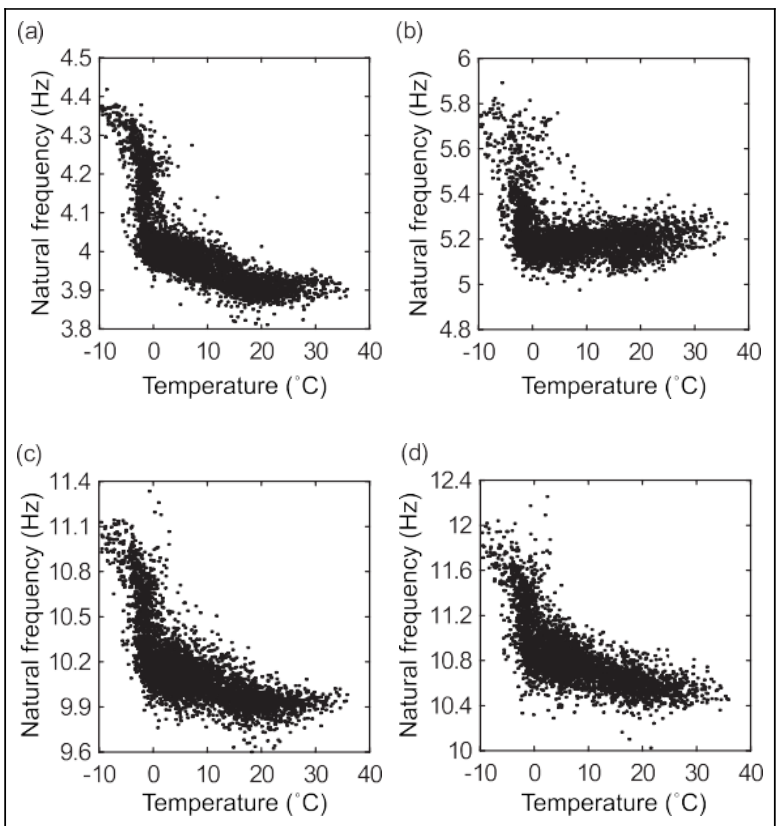

Figure 14. Graph of temperature versus the Z24 Bridge's (a) first natural frequency, (b) second natural frequency, (c) third natural frequency, and (d) fourth natural frequency.

temperatures above $08 \mathrm{C}$ to take into account this nonlinear effects. It was decided that the baseline will be made up of 16 observations, with 8 observations obtained from the day having the lowest temperature and 8 observations from the day having the highest temperature so that the relationship between the low or high temperatures and the natural frequencies can be captured.

It should be noted that in the creation of the baseline of the undamaged structure, the ambient temperature parameter is used because in the literature, it was found that when compared to other environmental conditions of the likes of humidity, temperature has a major influence on the vibration properties of bridges. And since PCA arranges the principal components in descending order, to make sure that the first principal component will account for an environmental effect, the temperature factor is chosen since it has the greatest effect on vibration properties when compared to other factor. The remaining environmental factors affecting the natural frequencies of the bridge will then be represented by the minor principal components. Hence, although the ambient temperature is used in the creation of the baseline, the proposed method will be able to separate the effects of other environmental conditions from damage effects since those environmental conditions will be represented by other minor principal components.

To get a rough indication on how many principal components are affected by choosing the two extreme 


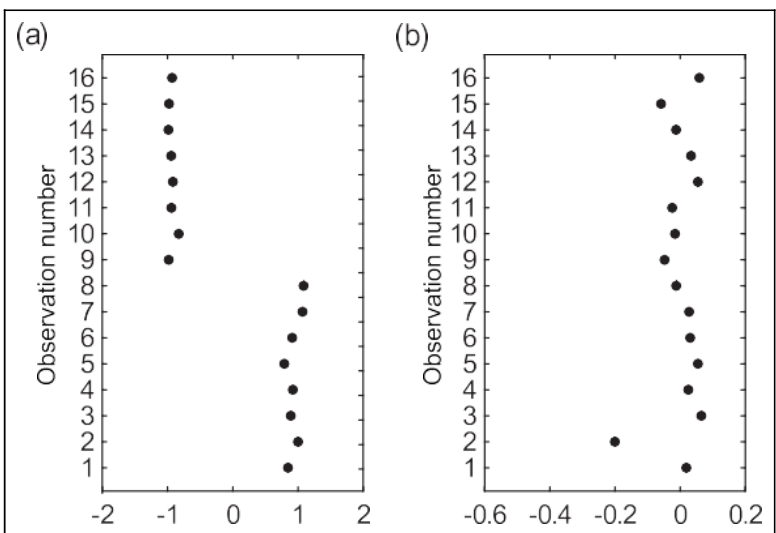

Figure 15. Graph of (a) first principal component and (b) second principal component for the baseline.

and opposite ambient temperatures to construct the baseline, PCA is first applied to the baseline data set alone (matrix $Z$ with dimensions 43 16). The first and second principal component plots are given in Figure 15. The high-temperature measurements in the first principal component plot cluster together on the lefthand side, while the low-temperature measurements cluster together on the right-hand side. Since two extreme and opposite temperature measurements have been chosen, this therefore indicates that the temperature variation affecting the data set is represented by the first principal component. In the second principal component plot (Figure 15(b)), the low- and hightemperature measurements cluster and mix together. The magnitude of the observations for that component is also relatively small when compared to the first principal component. This second principal component may represent other factors affecting the extreme cases in a similar way of the likes of other environmental conditions (e.g. humidity) or some nonlinear effects and noise. The way the baseline observations cluster and the magnitude of the observations in the principal components are noted for further comparison when the damage detection method is applied for monitoring.

After the baseline has been formulated, each subsequent measurement can be added to the baseline data set one at a time to give a 4317 matrix (matrix Z), and PCA be applied to obtain the principal components (to obtain matrix $\mathrm{Y}$ of equation (2)). To verify that the method does not alert damage when the structure is undamaged, and that it alerts damage when the structure is damaged, all the undamaged and damaged observations recorded are tested. Plots of results (first and second principal component plots only) of three randomly chosen undamaged cases and three randomly chosen damaged cases are given in Figures 16 and 17, respectively.
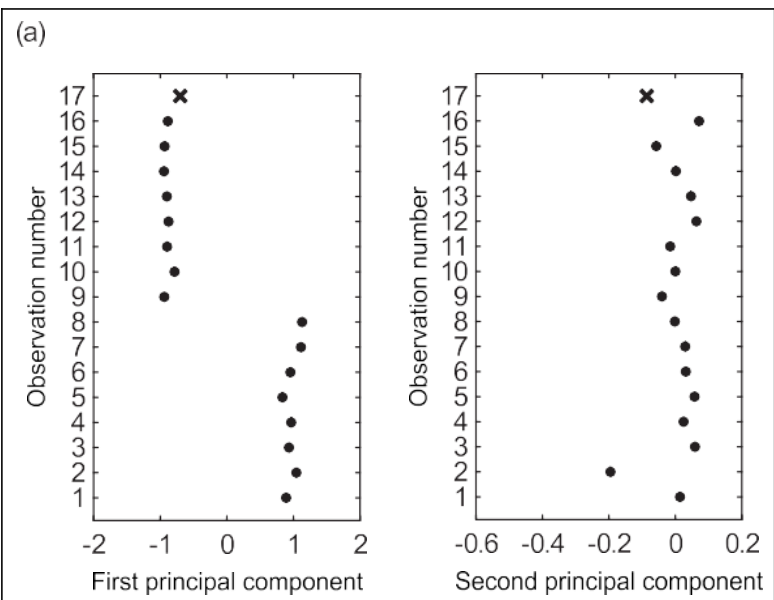

(b)
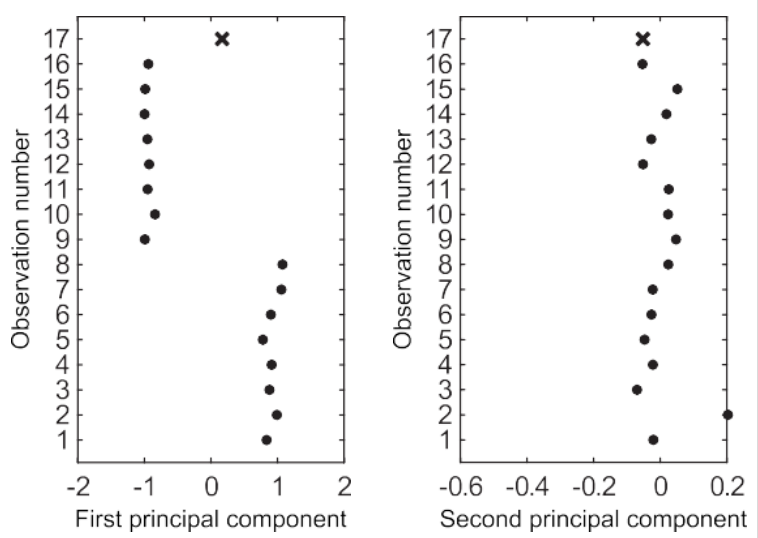

(c)
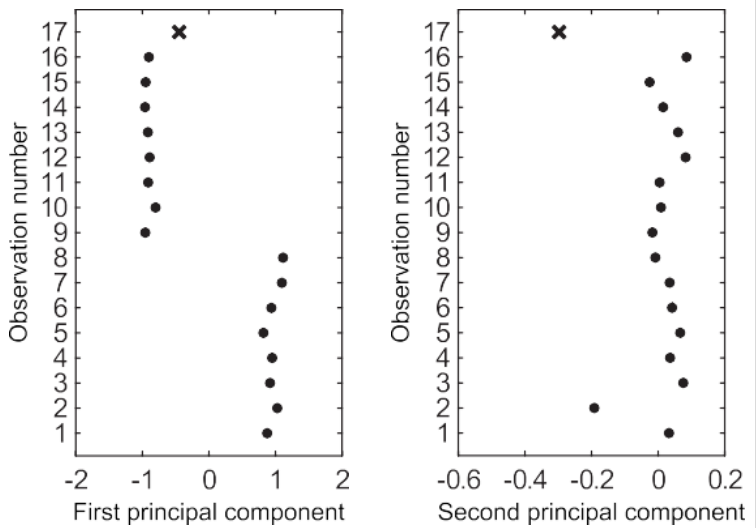

Figure 16. Graph of first and second principal components for randomly chosen undamaged (a) case 1, (b) case 2, and (c) case 3 .

From the first principal component plots given in Figures 16 and 17, it can be seen that the low- and high- temperature measurements for the two extreme cases used as baseline have a clear distinction between them, while they are mixed together in the second principal component plots. Hence, the second principal 


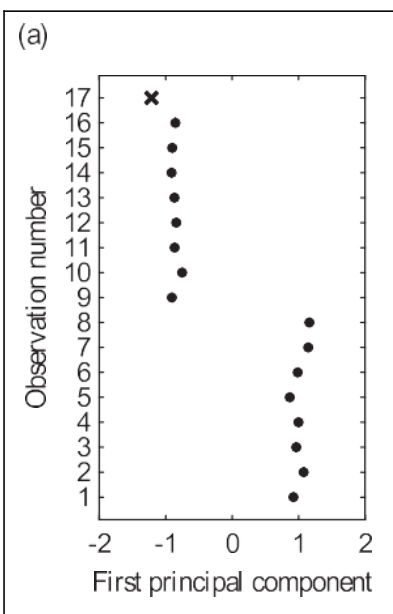

(b)

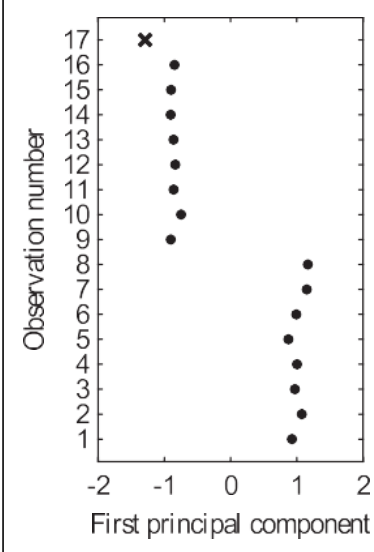

(c)
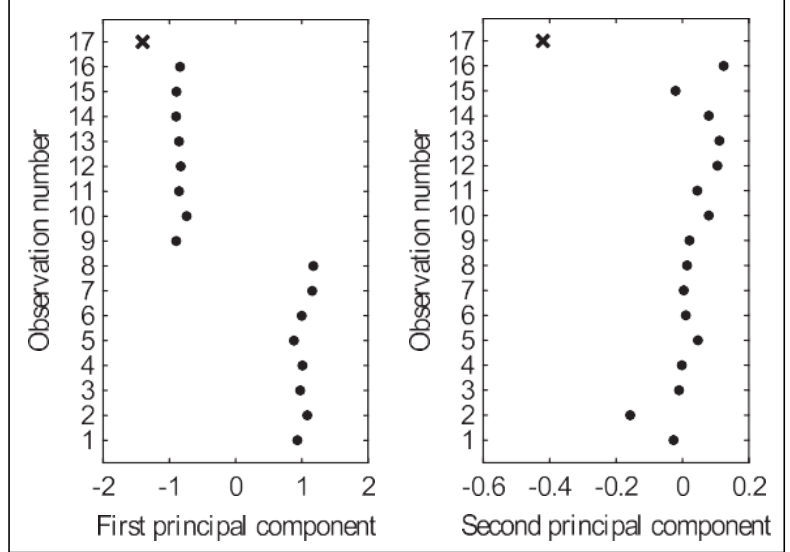

Figure 17. Graph of first and second principal components for randomly chosen damaged (a) case 1, (b) case 2, and (c) case 3.

component is discarded and only the first principal component plots are analyzed to distinguish damage effects from environmental effects. For the undamaged cases (Figure 16), the monitored cases lie between the two baselines in the first principal component plots. Thus, it is concluded that the structure is undamaged

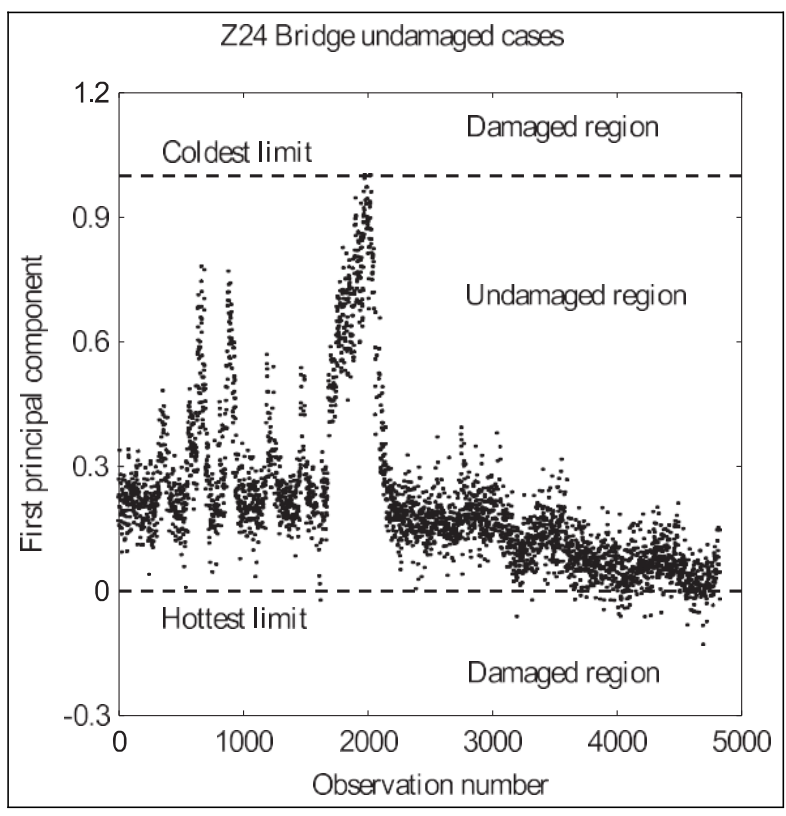

Figure 18. Results of the first principal component for all undamaged cases observation.

and the only significant factor affecting the data set is the temperature variation. The different temperature conditions the bridge was faced with from case to case can also be seen from the plots. For the damaged cases (Figure 17), the first principal component plots show that the monitored cases lie outside the baselines. This indicates that another factor(s) other than the temperature variation affects the data set significantly. This is attributed to damages which were introduced to the bridge during the monitoring period. Thus, damaged alert is raised for these damaged cases. The first principal component for the undamaged cases represents the temperature factor, while for the damaged cases, it represents the undamaged and damaged effects affecting the data set, and this is due to the fact that the damaged and undamaged effects produce a greater variance in the data set than the temperature effects.

To give a better representation of the results obtained for all the cases, plots of the first principal component for all the undamaged cases and damaged cases are given in Figures 18 and 19, respectively. All the cases are adjusted to have baseline values of 0 (high temperature) and 1 (low temperature). It should be noted that the plots give only the monitored cases being analyzed. The two horizontal dotted lines in the plots represent the baselines. It can be seen from both figures that most of the cases being analyzed are well classified. The successful rate of alerting damage for the damaged bridge is $98.0 \%$ while alerting undamaged when the bridge is undamaged is $97.8 \%$. Some undamaged cases 


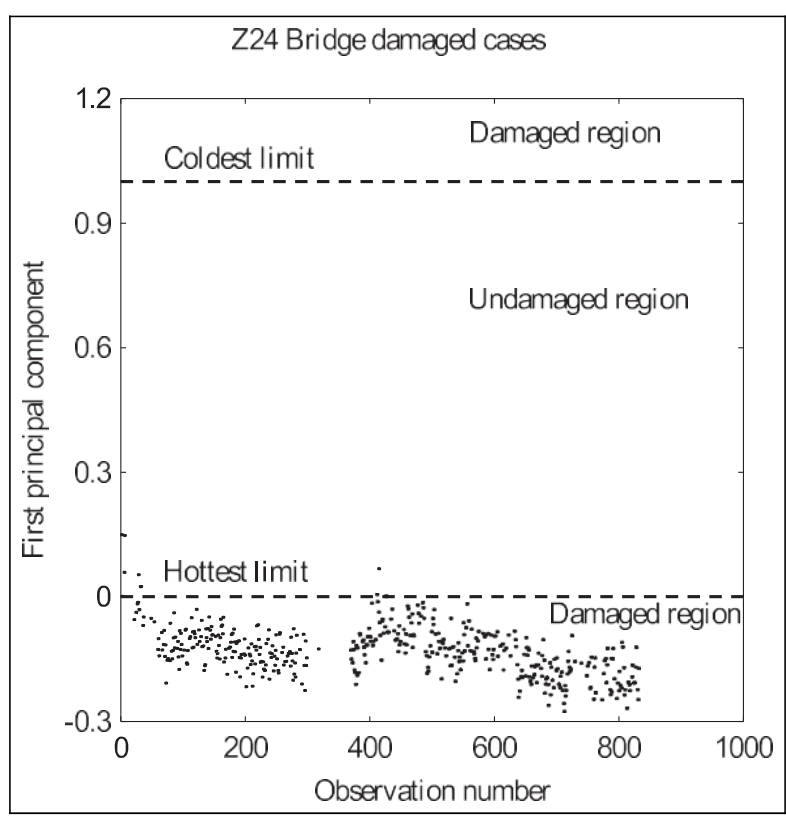

Figure 19. Results of the first principal component for all damaged cases observation.

are not well classified using the proposed method, and this may be attributed to the time lag in which the temperature of the whole bridge's materials change. The baseline of the undamaged structure was constructed with the help of the ambient temperature measurements. However, it is the temperature of the materials of the bridge that determines the vibration properties of the structure. Thus, if there was a time lag in which the temperature of the materials of the bridge change, the actual baseline used may not represent the real extreme cases. The two extreme temperature cases of the materials might have happened several hours later, thus the observations used in the baseline might not be those that should be used. Therefore, some of the undamaged cases will lie outside the baseline as can be seen in Figure 18.

From the results given in Figure 18, the temperature variations the structure was faced with can be seen clearly. The damage evolution can also be observed using the proposed method as can be observed from Figure 19. Moreover, from the results obtained, it can be seen that even though the ambient temperature was used in the selection of the baseline, this did not affect the performance of the proposed method to distinguish between damaged effects and all the environmental effects (e.g. humidity) affecting the natural frequencies of the bridge structure. It can therefore be said that this method proves its robustness in analyzing near real time this real-life bridge structure which was subjected to varying environmental conditions which had nonlinear effects on the damage sensitivity features.

\section{Conclusion}

A damage detection method is developed in this article to analyze structures that are subjected to varying environmental conditions. An approach to create the baseline of the undamaged structure, which consists of damage sensitivity features obtained at two extreme and opposite environmental conditions, is proposed. To allow near real-time monitoring of structures, subsequent measurements can be added to the baseline one at a time to create different data sets, and PCA is adopted for data processing. Analyzing the first few principal components of each data set, damage effects can then be distinguished from the effects of the varying environmental conditions. To decide on the number of principal components to be analyzed, it is proposed to analyze only the principal components that have the two extreme and opposite baseline observations to be separated from each other. The principal component that has the two extreme baseline observations to be mixed together should be discarded as well as the following components.

The proposed method is tested and validated using a numerical truss structure subjected to a varying temperature to simulate a varying environmental condition and the Z24 Bridge in Switzerland. The results demonstrate the ability of the proposed method in distinguishing damage effects from environmental effects affecting the damage sensitivity features of the structures. Damage evolution as well as the environmental conditions the structures are faced with can also be obtained from the proposed method. The method also proves its robustness in analyzing structures having damage sensitivity features which have nonlinear relationship with the environmental conditions in which they are obtained. The high successful rates obtained, show that the method does not require the baseline to consist of damage sensitivity features obtained from a wide range of environmental conditions. After the baseline is created, the environmental measurements are not required anymore to perform the proposed method, which, decreases the risk of failure of the SHM system. Another positive aspect of the proposed method is that, the control limits of the baseline created from the undamaged structure, is dictated by the extreme environmental conditions used. This, as a result, removes the needs of the engineers to make judgments on which control limits to adopt for damage detection. Also, even though the temperature measurements have been used for the creation of the baseline, other environmental factors of the likes of humidity and wind have been well separated from damaged effects using the proposed method as can be seen from the Z24 Bridge case study. Moreover, since the method allows the monitored cases to be analyzed one at a time, thus, this 
method can be used for near real-time monitoring of structures. The two possible factors that hinder realtime monitoring are (1) the time required to gather the damage sensitivity features and (2) the amount of time to perform the analysis. Due to all these aforementioned positive aspects of the proposed method, it can be concluded that the proposed method is a step forward for the implementation of real-time damage detection of real-life civil structures.

However, the proposed method has the limitation that it requires the baseline to consist of damage sensitivity features obtained at two extreme and opposite environmental conditions. This can sometimes be difficult to obtain. Moreover, the method requires the baseline to be selected using one environmental parameter which can lead to difficulties in choosing which environmental parameter to use since different environmental parameters may lead to different baselines as was discussed in the Z24 Bridge case study. Further work is on course to develop an approach to create the extreme cases baseline which will not be reached by the structure under its normal environmental conditions without using measurements of the environmental parameters so as to ensure that all the monitored observations in their undamaged status will lie inside the baseline.

\section{Acknowledgements}

The authors thank Professors Guido De Roeck and Edwin Reynders for providing the data of the Z24 Bridge.

\section{Declaration of conflicting interests}

The author(s) declared no potential conflicts of interest with respect to the research, authorship, and/or publication of this article.

\section{Funding}

This work was supported by the National Science Foundation of China (NSFC5160050924) and Zhejiang Qianjiang Talent Scheme (QJD1402009).

\section{References}

1. Sohn H. Effects of environmental and operational variability on structural health monitoring. Philos T Roy Soc A 2007; 365(1851): 539-560.

2. Farrar CR, Doebling SW, Cornwell PJ, et al. Variability of modal parameters measured on the Alamosa Canyon Bridge. In: Proceedings of the 15th international modal analysis conference, Orlanda, FL, 3-6 February 1997, pp. 257-263. Bethel, CT: Society for Experimental Mechanics.

3. Alampalli $S$. Influence of in-service environment on modal parameters. In: Proceedings of the 16th international modal analysis conference, Santa Barbara, CA, 2-5
February 1998, pp. 111-116. Bethel, CT: Society for Experimental Mechanics.

4. Peeters B and De Roeck G. One-year monitoring of the Z24-bridge: environmental effects versus damage events. Earthq Eng Struct Dyn 2001; 30(2): 149-171.

5. Rohrmann RG, Baessler M, Said S, et al. Structural causes of temperature affected modal data of civil structures obtained by long time monitoring. In: Proceedings of the 18th international modal analysis conference, San Antonio, TX, 7-10 February 2000, pp. 1-7. Bethel, CT: Society for Experimental Mechanics.

6. Ko JM, Chak KK, Wang JY, et al. Formulation of an uncertainty model relating modal parameters and environmental factors by using long-term monitoring data. In: Proceedings of smart structures and materials 2003: smart systems and nondestructive evaluation for civil infrastructures, San Diego, CA, 2-6 March 2003, pp. 298-307. Bellingham, WA: SPIE.

7. He X, Conte JP, Fraser M, et al. Long-term monitoring of a highway bridge. In: Proceedings of the 3rd international operational modal analysis conference, Portonovo, 4-6 May 2009, pp. 29-36. Red Hook, NY: Curran \& Associates Inc.

8. Ko $\mathbf{J}$ and Ni Y. Technology developments in structural health monitoring of large-scale bridges. Eng Struct 2005; 27(12): 1715-1725.

9. Zhou GD and Yi TH. A summary review of correlations between temperatures and vibration properties of longspan bridges. Math Probl in Eng 2014; 2014: 638209.

10. Fritzen C, Mengelkamp $G$ and Guemes A. Elimination of temperature effects on damage detection within a smart structure concept. In: Proceedings of the 4th international workshop on structural health monitoring, Stanford, CA, 15-17 September 2003, pp. 1530-1538. DEStech Publications Inc.

11. Dervilis N, Worden K and Cross E. On robust regression analysis as a means of exploring environmental and operational conditions for SHM data. J Sound Vib 2015; 347: 279-296.

12. Worden K, Sohn H and Farrar C. Novelty detection in a changing environment: regression and interpolation approaches. J Sound Vib 2002; 258(4): 741-761.

13. Peeters B, Maeck J and De Roeck G. Dynamic monitoring of the Z24-bridge: separating temperature effects from damage. In: Proceedings of the European COST F3 conference on system identification and structural health monitoring, Madrid, June 2000, pp. 377-386. Spain: Universidad Polite'cnica de Madrid.

14. Ni YQ, Fan K, Zheng G, et al. Automatic modal identification of cable-supported bridges instrumented with a long-term monitoring system. In: Proceedings of the smart structures and materials 2003: smart systems and nondestructive evaluation for civil infrastructures, San Diego, CA, 2-6 March 2003, pp. 329-339. Bellingham, WA: SPIE.

15. Kullaa J. Elimination of environmental influences from damage-sensitive features in a structural health monitoring system. In: Proceedings of the 1st European workshop 
on structural health monitoring, Paris, 10-12 July 2002, pp. 742-749. DEStech Publications Inc.

16. Yan AM, Kerschen G, De Boe P, et al. Structural damage diagnosis under varying environmental conditionspart I: a linear analysis. Mech Syst Signal Pr 2005; 19(4): 847-864.

17. Kullaa J. Structural health monitoring under variable environmental or operational conditions. In: Proceedings of the 2nd European workshop on structural health monitoring, Munich, 7-9 July 2004, pp. 1262-1269. DEStech Publications Inc.

18. Deraemaeker A, Reynders E, De Roeck G, et al. Vibration-based structural health monitoring using output-only measurements under changing environment. Mech Syst Signal Pr 2008; 22(1): 34-56.

19. Cross EJ, Worden K and Chen Q. Cointegration: a novel approach for the removal of environmental trends in structural health monitoring data. P Roy Soc Lond A Mat 2011; 467(2133): 2712-2732.

20. Manson G. Identifying damage sensitive, environment insensitive features for damage detection. In: Proceedings of the 3rd international conference on identification in engineering systems, Swansea, 15-17 April 2002, pp. 187-197. University of Swansea.
21. Yan AM, Kerschen G, De Boe P, et al. Structural damage diagnosis under varying environmental conditionspart II: local pca for non-linear cases. Mech Syst Signal Pr 2005; 19(4): 865-880.

22. Sohn H, Worden K and Farrar CR. Statistical damage classification under changing environmental and operational conditions. J Intell Mater Syst Struct 2002; 13(9): 561-574.

23. Reynders E, Wursten G and De Roeck G. Output-only structural health monitoring in changing environmental conditions by means of nonlinear system identification. Struct Health Monit 2014; 13(1): 82-93.

24. Smith LI. A tutorial on principal components analysis. Ithaca, NY: Cornell University, 2002, http://www.cs. otago.ac.nz/cosc453/student_tutorials/principal_com ponents. pdf

25. Sharma S. Applied multivariate techniques. New York: John Wiley \& Sons, 1996.

26. Krämer C, De Smet C and De Roeck G. Z24 bridge damage detection tests. In: Proceedings of the 17th international modal analysis conference, Kissimmee, FL, 8-11 February 1999, pp. 1023-1029. Bethel, CT: Society for Experimental Mechanics. 